Reviews in Digital Humanities • Vol. 2, No. 3

\title{
Review: Media History Digital Library
}

Nina Gary ${ }^{1}$, Becky Yatsuknenko ${ }^{1}$

${ }^{1}$ University of Texas at Austin

Published on: Mar 08, 2021

DOI: $10.21428 / 3 e 88 f 64 f .5 a f a a 9 a 7$

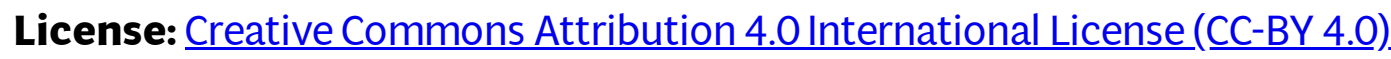




\section{Project}

Media History Digital Library

Project Director

Eric Hoyt, University of Wisconsin-Madison

\section{Project URL}

https://mediahistoryproject.org $L$

\section{Project Reviewers}

Nina Gary, University of Texas at Austin

Becky Yatsuknenko, University of Texas at Austin

\section{Project Overview}

\section{Eric Hoyt}

Over the past decade, the Media History_Digital Library_(MHDL). has transformed the study of film and broadcasting history, offering broad public access to over 2.5 million pages of out-of-copyright books and magazines. Prior to the MHDL's arrival, scholars had long utilized trade papers, such as Moving Picture World and Film Daily, to research the development of media industries and their impact upon audiences and culture. Yet, access to those sources remained limited. Scholars depended upon incomplete and low-quality microfilm facsimiles. The majority of film and broadcasting magazines, which had never been transferred to microfilm, were only available at a handful of institutions. And neither the microfilm nor print copies were full-text searchable. Instead, researchers had to turn pageby-page to look for relevant items to their research questions.

The MHDL was founded in 2009 by David Pierce, who stepped down from the project in 2017 to serve as the Assistant Chief of the Library of Congress National Audio-Visual Conservation Center. Pierce knew the breadth of extant film and broadcasting publications, and he knew the institutions and private collectors who possessed original copies (not simply the microfilm). He found generous individuals willing to fund the digitization of important magazines and borrowed from the collections of the Museum of Modern Art (MoMA), Pacific Film Archive, and private collectors. Pierce also possessed 25 years of experience investigating the copyright status of books and films. Whereas most Hollywood movies from the 1920s through the 1950s were still under copyright protection, most of the trade papers and fan magazines of this period belonged in the public domain. This has enabled the MHDL to share its collections on an open-access basis without a single copyright dispute. 
The MHDL's current director, Eric Hoyt, joined the project in 2011 to lead the project's data curation and software development initiatives. The Internet Archive agreed to serve as the scanning vendor and data hosting service, granting the MHDL its own sub-collection (accessible at http://archive.org/details/mediahistory). When Hoyt accepted a faculty position at University of Wisconsin-Madison in 2012, he brought the software development projects to the university, where he had been collaborating with Wendy Hagenmaier and Carl Hagenmaier. In 2013, Hoyt and his team launched Lantern, a search platform for the MHDL. In 2014, Hoyt and Charles Acland at Concordia University received a $\$ 200,000$ Digging into Data grant, sponsored by IMLS and SSHRC, to build Arclight-a data analytics platform that searches for trends across the MHDL's collection. The MHDL's platform improvements have also received $\$ 10,000$ in funding from the Mary Pickford Foundation and approximately $\$ 100,000$ from the University of Wisconsin-Madison, which became the official home of the project in 2017.

Despite the above-mentioned accomplishments, the MHDL's ability to fully engage its users has remained limited due to its data model, user interface, and dearth of digitized content published outside the United States. To remedy these limitations, the MHDL obtained a \$150,000 ACLS Digital Extension Grant to develop a new backend and frontend for the collection that will support the entry of granular metadata, including tags and other contributions from users, as well as sharing linked data with the American Film Institute (AFI) Catalog. Beyond these significant technical improvements, the "Globalizing and Enhancing the Media History Digital Library" initiative is enhancing the global scope of the collection through collaboration with a diverse and international group of scholars. The work on this initiative is ongoing, and it will reach its completion in mid-2021.

By enabling free access and searchability for both canonical media periodicals (e.g. Variety, Photoplay) and lesser known works (e.g. Camera!, Film Mercury), the MHDL and its discovery platforms, Lantern and Arclight, have enabled ambitious research projects and the production of new knowledge. For its contributions to the research landscape, the MHDL has been recognized with major awards from the Society for Cinema and Media Studies (SCMS), International Association for Media and History, and American Association of School Librarians. Every month, over 10,000 users from around the world run searches on Lantern, with the average user spending 10 minutes and 34 seconds per visit.

\section{Project Review}

Nina Gary and Becky Yatsuknenko

Led by Eric Hoyt of the University of Wisconsin-Madison, the Media History Digital Library (MHDL) offers free public access to over 2.5 million pages of film and media-related magazines and books in the public domain. Founded in 2009, MHDL includes "canonical media periodicals," like Variety and 
Photoplay, as well as collections contributed by MoMA, the Pacific Film Archive, and other larger institutions. Prior to the creation of MHDL, media periodicals were often only available in poor quality microfilm copies or, in many cases, in a small number of archives. MHDL has created a technologically and scholastically accessible site, making it an exemplary addition to digital archiving and digital humanities. It assists researchers in identifying and using media-related materials that would previously have required physical access.

The technical infrastructure of MHDL is trifurcated. The main website is built using HTML, CSS, JavaScript, and Bootstrap. The site's home page features links to the entire library of collections, an "About" page, Arclight, Lantern, and a page for teachers and students that includes potential lesson plans and assignments. Lantern, developed by the project team, allows users to search, visualize, and browse the MHDL collections. Lantern is a compilation of the open source Solr search engine, the Blacklight Ruby gem, and the JQuery Mix it Up Library. Arclight, also built by project team members, allows the results of Lantern searches to be turned into graphs that allow users to analyze trends within the corpus. ArcLight queries the Solr engine through a JavaScript frontend. Underlying all three technology stacks is the Internet Archive, which provides access to the digitized collections as well as its Internet Archive Bookbuilder, which display the works with "flippable" pages. As with many digital humanities projects, a host of other technologies patch together these main code bases to make a functional project.

As MHDL stands now, these platforms are still rather unfriendly interfaces that split off from the main site. The project team itself notes that the trifurcated structure with its underlying data model is both "decentralized" and "messy." Their plans for repairing the data model, technical separation, and the data's alignment to RDF and linked open data standards will undoubtedly serve users well in the future. The current focus of how to create a friendlier interface, improve the site's searchability, and publish more diverse content from non-U.S. sources is quite welcome. Even without those pending improvements MHDL sets itself apart from other digital archives in that it gives itself openly to pedagogical use, going so far as to create sample assignments written by educators involved in MHDL's creation. There are some twelve assignments listed in the section for educators, in addition to tutorials about how to navigate the site in the section for students. Few other digital archives make the specialized effort to advertise themselves to classroom-specific use. MHDL's decision to do so opens the door for future digital humanities projects, proving that there is funding for and interest in a site that has specific pedagogical intentions.

MHDL's acknowledgement of and plan to remedy the site's problems is a testament to their commitment to opening up the archive to all researchers, educators, and students alike. Though it is not in a nascent stage, the field of digital archiving has grown significantly since the site's founding in 
2009. In using the improved technologies designed for digital archives, MHDL has the potential to serve as a great model for future digital archives and beyond. 